\title{
Finite-Time Antisynchronization and Parameters Identification of Nonlinear Coupled Multiple-Weight Markovian Switching Complex Networks with Stochastic Perturbations
}

\author{
Haifeng Qiu (D), Liguo Weng, Bin Yu, and Yanghui Zhang \\ State Grid Hangzhou Xiaoshan Power Supply Company, Hangzhou 311200, China \\ Correspondence should be addressed to Haifeng Qiu; 13857177475@sina.cn
}

Received 15 May 2020; Revised 3 November 2020; Accepted 24 November 2020; Published 11 December 2020

Academic Editor: Maria L. Gandarias

Copyright (c) 2020 Haifeng Qiu et al. This is an open access article distributed under the Creative Commons Attribution License, which permits unrestricted use, distribution, and reproduction in any medium, provided the original work is properly cited.

\begin{abstract}
The paper mainly focuses on the synchronization of multiple-weight Markovian switching complex networks under nonlinear coupling mode. Based on the finite-time stability theory, Itô's lemma, and some inequality technologies, the synchronization criterion of network models in the nonlinear coupling mode is obtained; at the same time, unknown parameters of networks are also identified by an effective controller. In addition, several corollaries are given to illustrate the general applicability of the control rules in the paper. Finally, two typical numerical simulations are given to prove the rationality and feasibility of theoretical analysis of network models.
\end{abstract}

\section{Introduction}

Complex networks, as an interdisciplinary combination of nonlinear systems, control theory, graph theory, physics, and mathematical theory, have attracted the attention of all sectors of society. In human social activities, complex networks are closely related to our lives. Among them, real physical networks (e.g., power networks, transportation networks, and Internet networks) and abstract networks (e.g., financial networks, media networks, and interpersonal networks) both belong to complex networks $[1,2]$. In the many dynamic behaviors of complex networks, synchronization is one of the representative and important collective behaviors on complex networks $[3,4]$. The phenomenon of the network synchronization means that the dynamic status of the network nodes gradually tends to be consistent through the interaction between dynamic systems. In [5], based on the Lyapunov functional method and pinning control technique, some sufficient conditions are derived to ensure the synchronization of the network. Synchronization of nonlinear complex networks with multiple time-varying delays is achieved in [6]. These literatures mentioned above are all about the synchronization within one network; this kind of synchronization is called inner synchronization. However, it is also very important to study the synchronization between two or more networks; this kind of synchronization is called outer synchronization. At present, many control strategies have been proposed and applied to the synchronization of complex networks, such as adaptive control [7], pinning control [8, 9], sliding control [10], and intermittent control [11]. In the paper, in order to realize the antisynchronization of the networks, the adaptive control method will be adopted.

It is well known that the coupling relationships of complex networks cannot be all linear in real life. In many cases, the value of the variable $x(t)$ cannot be directly observed, and the observed value can only be $h(x(t))$. At this time, the nonlinear relationship $h(x(t))$ is used to achieve complex network synchronization [12]. Therefore, the nonlinear coupling synchronization problem also has great research value, and the results are even more practical. In [13], synchronization of nonlinear and delayed coupled neural networks with multiweights is achieved through pinning control technology. Based on the Lyapunov stability theory, synchronization 
problem of a nonlinear complex network with multiple timevarying delays is investigated in [6]. In addition, the topology of the network also has an important effect on the synchronization of nonlinear coupled complex networks.

In most of the networks considered above, researchers assume that the topology of the network is fixed, but this requirement is very strict in practical applications and only reflects ideal situations. In actual conditions, due to machine failure or maintenance, stochastic disturbances, and other reasons, the topology of the actual network will always change with time varying [14]. It is found through research that this random switching may depend on the Markovian process. In [15], finite-time synchronization of a class of nonlinear coupled Markovian jump time delay complex networks with stochastic noises is achieved via pinning control technology. In [16], by constructing a novel stochastic Lyapunov-Krasovskii function and employing the linear matrix inequalities (LMIs), Liu et al. studied cluster synchronization problem of Markovian switching complex networks with hybrid couplings. Furthermore, in order to research the complex network closer to the actual system, the unknown parameters of the Markovian switching complex network should be paid more attention [17]. This is because the synchronization process of the network may be affected or even disrupted by these unknown parameters.

In the real world, a number of networks can be modeled by complex dynamic networks with multiple weights, such as power networks, social networks, and communication networks. Each node in these networks is connected by multiple weights. In other words, there are multiple forms of coupling between nodes in multiple-weight networks. In the following, we take a power network in Figure 1 as an example to build a complex network with multiple weights. If the power network is viewed as a collection of many high-voltage transmission lines and various power stations, then all kinds of high-voltage transmission lines can be called "edge," and the connection points of these high-voltage transmission lines (transmission or consuming power stations, substations that distribute power) can be called "node," so that the power system is abstracted as a complex network in the general sense. On the other hand, power transmission lines can transmit power through high-voltage direct current, flexible axial current, and AC/DC hybrid. Because each transmission method has different weights, a complex dynamic network model with multiple weights can describe the power network more accurately. From the above analysis, it is very necessary and valuable to study complex dynamic networks with multiple weights. In [18], the author establishes a global synchronization criterion for a multiweighted public transport route network. In [19], the author considered the global synchronization problem of multiweight urban transport networks. Moreover, it should be noted that the time of the network synchronization is very important in the actual system.

In the actual engineering field, the time of the network synchronization is concerned by people, and scholars are eager to know the time of network synchronization. The finite-time control technology can realize this idea. In fact, finite-time synchronization means the optimal convergence time and finite-time control scheme can better reflect the robustness of the network $[20,21]$. Hence, it is very valuable to study the finite-time synchronization of complex networks. In [14], based on an M-matrix technique and stochastic analysis technique, synchronization of Markovian jumping complex networks was achieved in a finite time. In [22], finite-time synchronization for Markovian jumping complex dynamical frameworks with hybrid couplings is studied. In addition, time delays and stochastic disturbances always exist in the actual networks, and they also have a great impact on the network synchronization process [23, 24]. Thus, stochastic disturbances should be considered in the network synchronization process.

Inspired by the above discussion, the paper mainly focuses on that the finite-time antisynchronization and parameter identification of a class of nonlinear coupled multipleweight Markovian switching complex networks with stochastic perturbations. To the best of our knowledge, until now, there are rarely studies on the finite-time antisynchronization of nonlinear coupled multiple-weight Markovian switching complex networks with unknown parameters. Therefore, the research has significance in terms of theory and practice.

This paper is organized as follows. Some notations and necessary conditions will be given in Section 2. In Section 3, finite-time antisynchronization analysis for nonlinear coupled multiple-weight Markovian switching complex networks is discussed. In Section 4, in order to prove the validity of the above theoretical analysis, two numerical examples are given. The conclusions are drawn in Section 5.

\section{Preliminaries}

In order to complete the theoretical proof, some important mathematical notations and necessary conditions are given in this section. Firstly, some important mathematical notations are introduced as follows. Define matrix $A^{T}$ (or $x^{T}$ ) as the transpose matrix of $A$ (or $x)$. Let $E$ represent mathematical expectations. The Kronecker product of matrix is represented by the notation $\otimes$. Let $\|x\|_{2}$ as the 2 -norm of $x . I_{n} \in R^{n \times n}$ is defined as the $n$-dimensional identity matrix. The maximum eigenvalue of the matrix $A$ is denoted by $\lambda_{\max }(A)$. Secondly, some necessary conditions are given below.

Assumption 1 ([25]). Assume that a Lipschitz condition is satisfied by noise intensity function $\sigma_{i}\left(t, e_{i}(t), r(t)\right)$, and there exists a constant $\rho_{i} \geq 0$ such that

$$
\operatorname{trace}\left[\sigma_{i}^{T}\left(t, e_{i}(t), r(t)\right) \sigma_{i}\left(t, e_{i}(t), r(t)\right)\right] \leq \rho_{i}(r(t)) e_{i}^{T}(t) e_{i}(t)
$$

Assumption 2 ([26]). Let $0<\gamma<1$ and $\xi>0$. Assume that there exists a continuous function $g:[0, \infty) \longrightarrow[0, \infty)$ with $g(0)>0$ such that, for any $0 \leq t_{1} \leq t_{2}$

$$
g(t)-g(u) \leq-\xi \int_{u}^{t}(g(s))^{\gamma} d s .
$$

Assumption 3 ([25]). For arbitrary $x(t) \in R^{n}$ and $y(t) \in R^{n}$, 


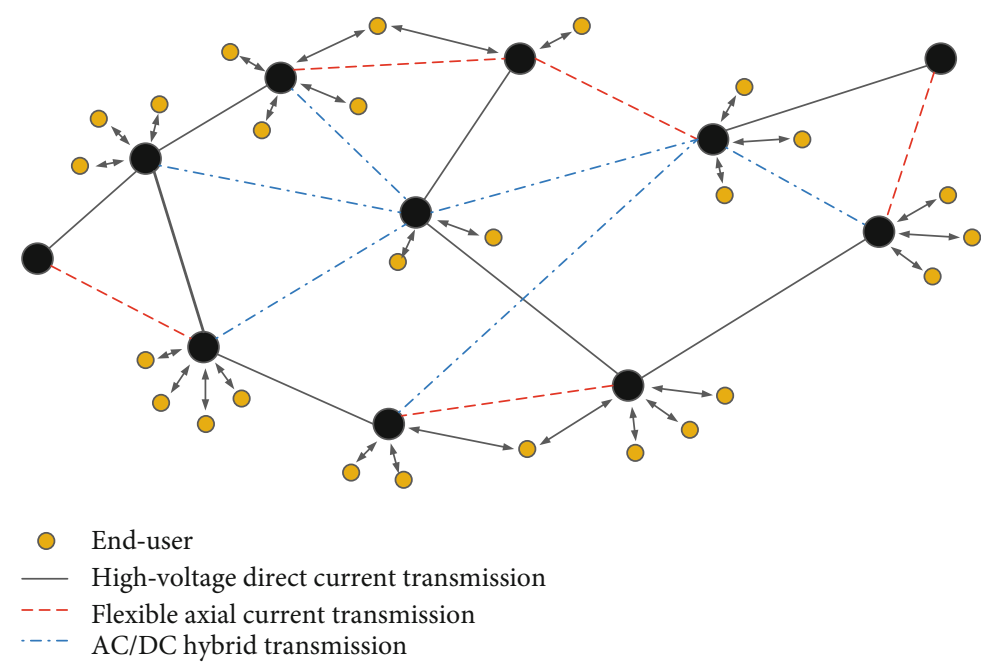

Figure 1: Power network diagram.

and nonlinear equation $f(t, x, \alpha)$ satisfies $f(t,-x, \alpha)=-f(t$, $x, \alpha)$, we can get

$$
\begin{gathered}
(x(t)+y(t))^{T}(f(t, x(t), \alpha)+f(t, y(t), \alpha)) \\
\leq(x(t)+y(t))^{T} v P(x(t)+y(t)),
\end{gathered}
$$

where $v$ is a nonnegative constant, and $P$ is a positive definite matrix.

Lemma 4 ([27]). Suppose that a continuous, positive definite function $V(t)$ satisfies the differential inequality $\dot{V}(t) \leq-\alpha$ $V^{\varepsilon}(t), \forall t \geq t_{0}, V\left(t_{0}\right) \geq 0$, where $\alpha>0,0<\varepsilon<1$. For any given $t_{0}, V(t)$ satisfies the inequality $V^{1-\varepsilon}(t) \leq V^{1-\varepsilon}\left(t_{0}\right)-\alpha(1-\varepsilon)$ $\left(t-t_{0}\right), t_{0} \leq t \leq t_{1}$, and $V(t) \equiv 0, \forall t \geq t_{1}$, we can get $t_{1}$

$$
t_{1}=t_{0}+\frac{V^{1-\varepsilon}\left(t_{0}\right)}{\alpha(1-\varepsilon)} .
$$

Lemma 5 ([27]). For any vectors $x(t), y(t) \in R^{n}$ and a positive definite matrix $K(K>0)$, the following inequality holds

$$
x^{T} y \leq \frac{1}{2}\left(x^{T} K^{-1} x+y^{T} K y\right)
$$

Lemma 6 ([28]). $b_{1}, b_{2}, \cdots, b_{n} \in R_{n}$ are any vectors, the following inequality holds

$$
\left|b_{1}\right|^{c}+\left|b_{2}\right|^{c}+\cdots+\left|b_{n}\right|^{c} \geq\left(\left|b_{1}\right|^{2}+\left|b_{2}\right|^{2}+\cdots+\left|b_{n}\right|^{2}\right)^{c / 2},
$$

where $c(0<c<2)$ is a real number.

\section{Finite-Time Antisynchronization between Two Nonlinear Coupled Double-Weight Markovian Switching Networks}

In the following, finite-time antisynchronization and identification between two nonlinear coupled double-weight
Markovian switching complex networks with stochastic perturbations are studied in this section.

3.1. Network Models. Consider the following multiple-weight Markovian switching complex network with $N$ nodes:

$$
\begin{aligned}
\dot{x}_{i}(t)= & f_{i 1}\left(x_{i}(t)\right)+f_{i 2}\left(x_{i}(t)\right) \alpha_{i}(t)+\sum_{j=1}^{N} c_{1} a_{i j}^{1}(r(t)) \Gamma_{1} x_{j}(t-\tau) \\
& +\sum_{j=1}^{N} c_{2} a_{i j}^{2}(r(t)) \Gamma_{2} h\left(x_{j}(t)\right), \quad i=1,2, \cdots, N
\end{aligned}
$$

where $x_{i}(t)=\left(x_{i 1}(t), x_{i 2}(t), \cdots, x_{i n}(t)\right)^{T} \in R^{n}$ is the state vector of the $i$ th node; $f_{i}: R^{n} \longrightarrow R^{n}$ and $f_{i 2}: R^{n} \longrightarrow R^{n \times n}$ are continuous vector function and matrix function, respectively; $h(\cdot): R^{n} \longrightarrow R^{n}$ is a nonlinear coupling function; $\alpha_{i}$ $(t)$ is an unknown parameter vector of the dynamic node; time delays $\tau$ satisfy $\tau>0 ; c_{d}\left(c_{d}>0, d=1,2\right)$ indicates the coupling strength for the $d$ th coupling form; $\Gamma_{d} \in R^{n \times n}(d$ $=1,2)$ is inner coupling matrix of the $d$ th coupling form $A^{d}(r(t))=\left(a_{i j}^{d}(r(t))\right)_{N \times N} \in R^{N \times N}(d=1,2) \quad$ is topological structure matrix in the $d$ th coupling form, which represents topological structure of the network at time $t$ in mode $r(t)$; $A^{d}(r(t))$ can be defined as if there exists a connection between node $i$ and node $j(j \neq i)$ for the $m$ th coupling form, then $a_{i j}^{d} \neq 0$; otherwise, $a_{i j}^{d}=0$. The diagonal elements of $A(r$ $(t))$ are defined as

$$
a_{i i}^{d}(r(t))=-\sum_{j=1, j \neq i}^{N} a_{i j}^{d}(r(t)), \quad d=1,2 ; i=1,2, \cdots, N .
$$

Let $\{r(t), t \geq 0\}$ be a right-continuous Markovian process in probability space, which takes values in a finite space $S=\{1,2 \cdots l\}$ with generator $\Pi=\left(\pi_{p q}\right)_{m \times m}$. The transition probability from the $p$ th mode at time $t$ to the $q$ th mode at 
time $t+\Delta t$ is defined as follow:

$$
P\{r(t+\triangle t)=q \mid r(t)=p\}=\left\{\begin{array}{l}
\pi_{p q} \triangle t+o(\triangle t), \quad \text { if } q \neq p, \\
1+\pi_{p p} \triangle t+o(\triangle t), \quad \text { if } q=p,
\end{array}\right.
$$

where $\Delta t>0, \lim _{\Delta t \rightarrow 0} o(\Delta t) / \Delta t=0$, and transition rate $\pi_{p q}\left(\pi_{p q}\right.$ $>0)$ satisfies

$$
\pi_{p p}=-\sum_{q=1, q \neq p}^{m} \pi_{p q}
$$

Network (7) is considered as drive system, then the response network as

$$
\begin{aligned}
\dot{y}_{i}(t)= & f_{i 1}\left(y_{i}(t)\right)+f_{i 2}\left(y_{i}(t)\right) \widehat{\alpha}_{i}(t)+\sum_{j=1}^{N} c_{1} b_{i j}^{1}(r(t)) \Gamma_{1} y_{j}(t-\tau) \\
& +\sum_{j=1}^{N} c_{2} b_{i j}^{2}(r(t)) \Gamma_{2} h\left(y_{j}(t)\right)+\sigma_{i}\left(t, e_{i}(t), r(t)\right) \dot{\omega}(t) \\
& +u_{i}(t), \quad i=1,2, \cdots, N,
\end{aligned}
$$

where $y_{i}(t)=\left(y_{i 1}(t), y_{i 2}(t), \cdots, y_{i n}(t)\right)^{T} \in R^{n}$ is the state vector of the $i$ th node; $\widehat{\alpha}_{i}(t)$ is estimation of the unknown parameter vector $\alpha_{i}(t) ; B^{d}(r(t))=\left(b_{i j}^{d}(r(t))\right)_{N \times N} \in R^{N \times N}(d=1,2)$ is the same as the definition of $A^{d}(r(t))$ above; $u_{i}(t)$ is a nonlinear controller; $\omega(t)=\left(\omega_{1}(t), \omega_{2}(t), \cdots, \omega n(t)\right)^{T}$ is the $n$-dimensional Weiner process. $\sigma_{i}\left(t, x_{i}(t), r(t)\right)$ is noise intensity function, which describes the random impact of the environment on the network.

Define the system synchronization error as follows:

$$
e_{i}(t)=x_{i}(t)+y_{i}(t), \quad i=1,2, \cdots, N,
$$

where $e_{i}(t)=\left(e_{i 1}(t), e_{i 2}(t) \cdots e_{i n}(t)\right)^{T}$ represents state error of $i$ th node.

Outer synchronization means that the behavior of corresponding nodes between two or more networks tends to be consistent. In other words, the error between the drive network and the response network is zero; the synchronization and identification of the system will be achieved.

Definition 7 . For an arbitrary $t\left(t>t_{1}>0\right)$, if each node of the network satisfies the following equation, then the drive system (7) and response system (11) can achieve synchronization in a finite time $t_{1}$.

$$
\lim _{t \rightarrow t_{1}} E\left\|e_{i}(t)\right\|=\lim _{t \rightarrow t_{1}} E\left\|x_{i}(t)+y_{i}(t)\right\|_{2}=0, \quad i=1,2, \cdots, N .
$$

Remark 8. When the nonlinear coupled double-weight Markovian switching complex network (7) and (11) gradually realizes antisynchronization at a finite time $t_{1}$, the unknown parameter vector $\alpha_{i}(t)$ will be identified, expression as

$$
\lim _{t \rightarrow t_{1}} \sum_{i=1}^{N}\left(\left\|\widehat{\alpha}_{i}(t)-\alpha_{i}\right\|_{2}\right)=0 .
$$

3.2. Main Results. In this part, finite-time antisynchronization between two nonlinear coupled double-weight Markovian switching complex networks is analyzed and proven through a designed nonlinear controller with update law.

From the network (7) and (11), the synchronization error of node dynamics system can be written as:

$$
\begin{aligned}
\dot{e}_{i}(t)= & \dot{x}_{i}(t)+\dot{y}_{i}(t)=f_{i 1}\left(x_{i}(t)\right)+f_{i 2}\left(x_{i}(t)\right) \alpha_{i}(t)+f_{i 1}\left(y_{i}(t)\right) \\
& +f_{i 2}\left(y_{i}(t)\right) \widehat{\alpha}_{i}(t)+\sigma_{i}\left(t, e_{i}(t), r(t)\right) \dot{\omega}(t) \\
& +\sum_{j=1}^{N} c_{1} a_{i j}^{1}(r(t)) \Gamma_{1} x_{j}(t-\tau) \\
& +\sum_{j=1}^{N} c_{2} a_{i j}^{2}(r(t)) \Gamma_{2} h\left(x_{j}(t)\right) \\
& \cdot \sum_{j=1}^{N} c_{1} b_{i j}^{1}(r(t)) \Gamma_{1} y_{j}(t-\tau) \\
& +\sum_{j=1}^{N} c_{2} b_{i j}^{2}(r(t)) \Gamma_{2} h\left(y_{j}(t)\right)+u_{i}(t) .
\end{aligned}
$$

Obviously, when the synchronization error (15) converges to zero in a finite time, the finite-time synchronization of networks will be achieved; at the same time, the unknown parameters of the system will be identified accordingly. Therefore, by designing an effective controller $u_{i}(t)$, the finite-time antisynchronization problem of the nonlinear coupled double-weight complex networks will be solved.

Theorem 9. Design the controller with adaptive law as follows

$$
\left\{\begin{array}{l}
u_{i}(t)=-k(r(t)) \operatorname{sign}\left(e_{i}(t)\right)\left|e_{i}(t)\right|^{\gamma}-\eta_{i}(r(t)) e_{i}(t)-\sum_{j=1}^{N} c_{1} a_{i j}^{1} r(t) \Gamma_{1} x_{j}(t-\tau)-\sum_{j=1}^{N} c_{1} b_{i j}^{1} r(t) \Gamma_{1} y_{j}(t-\tau)+\sum_{j=1}^{N} c_{2}\left(a_{i j}^{2} r(t)-b_{i j}^{2} r(t)\right) \Gamma_{2} h\left(y_{j}(t)\right), \\
\dot{\hat{\alpha}}_{i}(t)=\lambda_{i}(r(t)) f_{i 2}^{T}\left(y_{i}(t)\right) e_{i}(t)-\varphi_{i}(r(t)) \operatorname{sign}\left(\widehat{\alpha}_{i}(t)\right)\left(\left|\alpha \wedge_{i}(t)\right|+M_{\alpha}\right)^{\gamma}, \quad i=1,2, \cdots, N
\end{array}\right.
$$


where control parameters $\eta_{i}(r(t))>0, k_{i}(r(t))>0, \lambda_{i}(r(t))$ $>0$, and $\varphi_{i}(r(t))>0(r \in S) ; M_{\alpha}$ is a known constant and satisfies the condition $\left|\widehat{\alpha}_{i}\right| \leq M_{\alpha}$, and if the following inequality holds

$$
\begin{gathered}
\sum_{q=1}^{m} \pi_{p q}(D(q)-Q(p)) \leq 0, \\
\widetilde{K}+\widetilde{A}+\tilde{\Omega}+\left(\frac{1}{2} \Theta(r(t))+\tilde{O}-\Xi(r(t))\right) \otimes I_{n} \leq 0,
\end{gathered}
$$

where $D(q)$ is a positive definite matrix of appropriate dimension, and $Q(q)$ is an arbitrary symmetric matrix. $\Theta(r(t))=$ $\operatorname{diag}\left\{\rho_{1}(r(t)), \rho_{2}(r(t)), \cdots, \rho_{N}(r(t))\right\} ; \quad \operatorname{sign}\left(e_{i}(t)\right)=\operatorname{diag}\{$ sign $\left(e_{i 1}(t)\right)$, sign $\left.\left(e_{i 2}(t)\right), \cdots, \operatorname{sign}\left(e_{i n}(t)\right)\right\} ; \tilde{A}=(1 / 2) c_{2}\left(A^{2} \otimes\right.$ $\left.\Gamma_{2}\right) K^{-1}\left(A^{2} \otimes \Gamma_{2}\right)^{T} ; \tilde{O}=L^{2}\|K\| I_{N} ; \omega_{p}=\sum_{q=1}^{m}(D(q)-Q(p)) / 2 ;$ $\tilde{\Omega}=v I_{N} \otimes P ; \widetilde{K}=I_{N} \otimes \omega_{p} ; \Xi(r(t))=\operatorname{diag}\left\{\eta_{1}(r(t)), \eta_{2}(r(t))\right.$, $\left.\cdots, \eta_{N}(r(t))\right\}$.

Then, by controller (16) and inequality condition (17), the antisynchronization between two nonlinear coupled double-weight Markovian switching complex networks (7) and (11) can be achieved in a finite time $t_{1}$, and according to Lemma 4 , the finite time $t_{1}$ can be expressed as follows:

$$
t_{1} \leq t_{0}+\frac{V\left(t_{0}, r\left(t_{0}\right)\right)^{1-\varepsilon}}{\psi 2^{\varepsilon}(1-\varepsilon)},
$$

where $V\left(t_{0}, r\left(t_{0}\right)\right)=\left((1 / 2) \sum_{i=1}^{N} e_{i}^{T}\left(t_{0}\right) e_{i}\left(t_{0}\right)\right)+\left((1 / 2) \sum_{i=1}^{N}(1 /\right.$ $\left.\left.\lambda_{i}\left(r\left(t_{0}\right)\right)\right) \tilde{a}_{i}^{T}\left(t_{0}\right) \tilde{a}_{i}\left(t_{0}\right)\right), \quad \psi=\min \left\{\min (k(r)), \xi / 2^{(1+\beta) / 2} \min \right.$ $(\varphi(r))\}$.

Proof. Construct the Lyapunov function as follows:

$$
\begin{aligned}
V(t, e(t), p)= & \frac{1}{2} \sum_{i=1}^{N} e_{i}^{T}(t) e_{i}(t)+\frac{1}{2} \sum_{i=1}^{N} \frac{1}{\lambda_{i}(p)} \tilde{\alpha}_{i}^{T}(t) \tilde{\alpha}_{i}(t) \\
& +\sum_{i=1}^{N} \int_{t-\tau}^{t} e_{i}^{T}(\theta) e_{i}(\theta) d \theta .
\end{aligned}
$$

According to Itô's lemma, stochastic stability theory, and differential operator $\mathscr{L}$ [29], we can get

$$
\begin{aligned}
\mathscr{L} V(t, e(t), p)= & \sum_{i=1}^{N} e_{i}^{T}(t) \dot{e}_{i}(t)+\sum_{i=1}^{N} \frac{1}{h_{i}(p)} \dot{\tilde{\alpha}}_{i}^{T}(t) \tilde{\alpha}_{i}(t) \\
& +\sum_{i=1}^{N} e_{i}^{T}(t) e_{i}(t)-\sum_{i=1}^{N} e_{i}^{T}(t-\tau) e_{i}(t-\tau) \\
& +\sum_{q=1}^{m} \pi_{p q} \sum_{i=1}^{N} \int_{t-\tau}^{t} e_{i}^{T}(\theta) e_{i}(\theta) d \theta \\
& +\sum_{q=1}^{m} \pi_{p q} \frac{1}{2} \sum_{i=1}^{N} e_{i}^{T}(t) e_{i}(t) \\
& +\frac{1}{2} \sum_{i=1}^{N} \operatorname{trace}\left[\sigma_{i}^{T}\left(t, e_{i}(t), p\right) \sigma_{i}\left(t, e_{i}(t), p\right)\right] .
\end{aligned}
$$

Bringing equation (15) into (20) we can get.

$$
\begin{aligned}
\mathscr{L} V(t, e(t), p)= & \sum_{i=1}^{N} e_{i}^{T}(t)\left\{f_{i 1}\left(x_{i}(t)\right)+f_{i 2}\left(x_{i}(t)\right) \alpha_{i}(t)+f_{i 1}\left(y_{i}(t)\right)\right. \\
& +f_{i 2}\left(y_{i}(t)\right) \widehat{\alpha}_{i}(t)+u_{i}(t)+\sum_{j=1}^{N} c_{1} a_{i j}^{1}(r(t)) \Gamma_{1} x_{j}(t-\tau) \\
& +\sum_{j=1}^{N} c_{2} a_{i j}^{2}(r(t)) \Gamma_{2} h\left(x_{j}(t)\right)+\sum_{j=1}^{N} c_{1} b_{i j}^{1}(r(t)) \Gamma_{1} y_{j}(t-\tau) \\
& \left.+\sum_{j=1}^{N} c_{2} b_{i j}^{2}(r(t)) \Gamma_{2} h\left(y_{j}(t)\right)+\sigma_{i}\left(t, e_{i}(t), r(t)\right) \dot{\omega}(t)\right\} \\
& +\sum_{i=1}^{N} \frac{1}{h_{i}(p)} \dot{\tilde{\alpha}}_{i}^{T}(t) \tilde{\alpha}_{i}(t)+\sum_{i=1}^{N} e_{i}^{T}(t) e_{i}(t) \\
& -\sum_{i=1}^{N} e_{i}^{T}(t-\tau) e_{i}(t-\tau)+\sum_{q=1}^{m} \pi_{p q} \sum_{i=1}^{N} \int_{t-\tau}^{t} e_{i}^{T}(\theta) D(q) e_{i}(\theta) d \theta \\
& +\sum_{q=1}^{m} \pi_{p q} \frac{1}{2} \sum_{i=1}^{N} e_{i}^{T}(t) D(q) e_{i}(t) \\
& +\frac{1}{2} \sum_{i=1}^{N} \operatorname{trace}\left[\sigma_{i}^{T}\left(t, e_{i}(t), p\right) \sigma_{i}\left(t, e_{i}(t), p\right)\right] \\
= & \sum_{i=1}^{N} e_{i}^{T}(t)\left\{f_{i}\left(t, x_{i}(t), \alpha_{i}\right)+f_{i}\left(t, y_{i}(t), \alpha_{i}\right)\right. \\
& -f_{i 2}\left(y_{i}(t)\right) \tilde{\alpha}_{i}+u_{i}(t)+\sum_{j=1}^{N} c_{1} a_{i j}^{1}(r(t)) \Gamma_{1} x_{j}(t-\tau) \\
& +\sum_{j=1}^{N} c_{2} a_{i j}^{2}(r(t)) \Gamma_{2} h\left(x_{j}(t)\right)+\sum_{j=1}^{N} c_{1} b_{i j}^{1}(r(t)) \Gamma_{1} y_{j}(t-\tau) \\
& \left.+\sum_{j=1}^{N} c_{2} b_{i j}^{2}(r(t)) \Gamma_{2} h\left(y_{j}(t)\right)+\sigma_{i}\left(t, e_{i}(t), r(t)\right) \dot{\omega}(T)\right\} \\
& +\sum_{i=1}^{N} \frac{1}{h_{i}(p)} \dot{\hat{\alpha}}_{i}^{T}(t) \tilde{\alpha}_{i}(t)+\sum_{i=1}^{N} e_{i}^{T}(t) e_{i}(t) \\
& -\sum_{i=1}^{N} e_{i}^{T}(t-\tau) e_{i}(t-\tau)+\sum_{q=1}^{m} \pi_{p q} \sum_{i=1}^{N} \int_{t-\tau}^{t} e_{i}^{T}(\theta) D(q) e_{i}(\theta) d \theta \\
& +\frac{1}{2} \sum_{i=1}^{N} \operatorname{trace}\left[\sigma_{i}^{T}\left(t, e_{i}(t), p\right) \sigma_{i}\left(t, e_{i}(t), p\right)\right] \\
& +\sum_{q=1}^{m} \pi_{p q} \frac{1}{2} \sum_{i=1}^{N} e_{i}^{T}(t) D(q) e_{i}(t), \\
&
\end{aligned}
$$

where $D(q)$ is a positive definite matrix with suitable dimension.

According to controller (16), Assumption 1, and Assumption 2, equation (21) can be written as

$$
\begin{aligned}
\mathscr{L} V(t, e(t), p)= & \sum_{i=1}^{N} e_{i}^{T}(t)\left\{-\eta_{i}(p) e_{i}(t)+f_{i}\left(t, x_{i}(t), \alpha_{i}\right)+f_{i}\left(t, y_{i}(t), \alpha_{i}\right)\right. \\
& -f_{i 2}\left(y_{i}(t)\right) \tilde{\alpha}_{i}+\sum_{j=1}^{N} c_{2} a_{i j}^{2}(p) \Gamma_{2}\left(h\left(x_{j}(t)\right)+h\left(y_{j}(t)\right)\right. \\
& \left.-k(p) \operatorname{sign}\left(e_{i}(t)\right)\left|e_{i}(t)\right|^{\gamma}\right\} \\
& -\varphi(p) \sum_{i=1}^{N} \frac{1}{\lambda_{i}} \operatorname{sign}\left(\widehat{\alpha}_{i}(t)\right)\left(\left|\alpha \wedge_{i}(t)\right|+M_{\alpha}\right)^{\gamma} \tilde{\alpha}_{i}(t) \\
& -\sum_{i=1}^{N} e_{i}^{T}(t-\tau) e_{i}(t-\tau)+\sum_{i=1}^{N} e_{i}^{T}(t) f_{i 2}\left(y_{i}(t)\right) \tilde{\alpha}_{i}(t)
\end{aligned}
$$




$$
\begin{aligned}
& +\sum_{q=1}^{m} \pi_{p q} \sum_{i=1}^{N} \int_{t-\tau}^{t} e_{i}^{T}(\theta) D(q) e_{i}(\theta) d \theta \\
& +\sum_{i=1}^{N} e_{i}^{T}(t) e_{i}(t)+\sum_{q=1}^{m} \pi_{p q} \frac{1}{2} \sum_{i=1}^{N} e_{i}^{T}(t) D(q) e_{i}(t) \\
& +\frac{1}{2} \rho_{i}(r(t)) e_{i}^{T}(t) e_{i}(t) \\
= & \sum_{i=1}^{N} e_{i}^{T}(t)\left\{f_{i}\left(t, x_{i}(t), \alpha_{i}\right)+f_{i}\left(t, y_{i}(t), \alpha_{i}\right)\right. \\
& -k(p) \operatorname{sign}\left(e_{i}(t)\right)\left|e_{i}(t)\right|^{\gamma}-\eta_{i}(p) e_{i}(t) \\
& \left.+\sum_{j=1}^{N} c_{2} a_{i j}^{2}(p) \Gamma_{2} H\left(e_{j}(t)\right)\right\}+\sum_{q=1}^{m} \pi_{p q} \frac{1}{2} \sum_{i=1}^{N} e_{i}^{T}(t) D(q) e_{i}(t) \\
& -\xi \sum_{i=1}^{N} \int_{t-\tau}^{t}\left(e_{i}^{T}(\theta) e_{i}(\theta)\right)^{(\gamma+1) / 2} d \theta \\
& +\sum_{q=1}^{m} \pi_{p q} \sum_{i=1}^{N} \int_{t-\tau}^{t} e_{i}^{T}(\theta) D(q) e_{i}(\theta) d \theta \\
& +\frac{1}{2} \rho_{i}(r(t)) e_{i}^{T}(t) e_{i}(t) \\
& -\varphi(p) \sum_{i=1}^{N} \frac{1}{\lambda_{i}(p)} \operatorname{sign}\left(\widehat{\alpha}_{i}(t)\right)\left(\left|\alpha \wedge_{i}(t)\right|+M_{\alpha}\right)^{\gamma} \tilde{\alpha}_{i}(t)
\end{aligned}
$$

where $H\left(e_{j}(t)\right)=h\left(x_{j}(t)\right)+h\left(y_{j}(t)\right)$.

According to Lemma 5, we can get

$$
\begin{aligned}
\sum_{j=1}^{N} c_{2} a_{i j}^{2}(p) \Gamma_{2} H\left(e_{j}(t)\right)= & c_{2} e^{T}(t)\left(A^{2} \otimes \Gamma_{2}\right) H(e(t)) \\
\leq & \frac{1}{2} c_{2} e^{T}(t)\left(A^{2} \otimes \Gamma_{2}\right) K^{-1}\left(A^{d} \otimes \Gamma_{d}\right)^{T} e(t) \\
& +\frac{1}{2} H^{T}(e(t))\|K\| H(e(t)) .
\end{aligned}
$$

In addition, for arbitrary $x(t), y(t) \in R^{n}$, if there exists a constant $L>0$, then following inequality will hold

$$
\|f(x(t))+f(y(t))\| \leq L\|(x(t)+y(t))\| .
$$

Then, we have

$$
H^{T}(e(t))\|K\| H(e(t)) \leq L^{2}\|K\| e^{T}(t) e(t) .
$$

Thus,

$$
\begin{aligned}
\sum_{j=1}^{N} c_{2} a_{i j}^{2}(p) \Gamma_{2} H\left(e_{j}(t)\right)= & c_{2} e^{T}(t)\left(A^{2} \otimes \Gamma_{2}\right) H(e(t)) \\
\leq & \frac{1}{2} c_{2} e^{T}(t)\left(A^{2} \otimes \Gamma_{2}\right) K^{-1}\left(A^{d} \otimes \Gamma_{d}\right)^{T} e(t) \\
& +\frac{1}{2} L^{2}\|K\| e^{T}(t) e(t) .
\end{aligned}
$$

Based on Lemma 6, we have

$$
\begin{aligned}
-\sum_{i=1}^{N} e_{i}^{T}(t) \operatorname{sign}\left(e_{i}(t)\right)\left|e_{i}(t)\right|^{\gamma} & =-\sum_{i=1}^{N} \sum_{j=1}^{N} e_{i j}(t)\left|e_{i j}(t)\right|^{\gamma} \operatorname{sign}\left(e_{i j}(t)\right) \\
& =-\sum_{i=1}^{N} \sum_{j=1}^{N}\left|e_{i j}(t)\right|^{1+\gamma} \\
& \leq-\left(\sum_{i=1}^{N} \sum_{j=1}^{N}\left|e_{i j}(t)\right|^{2}\right)^{1+\gamma} \\
& =-\left(\sum_{i=1}^{N} e_{i}^{T}(t) e_{i}(t)\right)^{(1+\gamma) / 2} .
\end{aligned}
$$

Due to $\left|\widehat{\alpha}_{i}\right| \leq M_{\alpha}$, and from some inequality, relationships can obtain

$$
\left|\tilde{\alpha}_{i}(t)\right|=\left|\widehat{\alpha}_{i}(t)-\alpha_{i}(t)\right| \leq\left|\widehat{\alpha}_{i}(t)\right|+\left|\alpha_{i}(t)\right| \leq\left|\widehat{\alpha}_{i}(t)\right|+M_{\alpha} .
$$

Thus,

$$
\begin{aligned}
& -\sum_{i=1}^{N}\left(\left|\alpha \wedge_{i}(t)\right|+M_{\alpha}\right)^{\gamma} \operatorname{sign}\left(\widehat{\alpha}_{i}(t)\right) \tilde{\alpha}_{i}(t) \\
& \quad=-\sum_{i=1}^{N}\left(\left|\alpha \wedge_{i}(t)\right|+M_{\alpha}\right)^{\gamma} \tilde{\alpha}_{i}(t) \leq-\sum_{i=1}^{N}\left|\alpha \wedge_{i}(t)-\alpha_{i}(t)\right|^{\gamma} \tilde{\alpha}_{i}(t) \\
& \quad=-\sum_{i=1}^{N}\left|\tilde{\alpha}_{i}(t)\right|^{\gamma+1} .
\end{aligned}
$$

According to Assumption 3, and bringing the inequality (23), (27), (29) into equation (22), we can get

$$
\begin{gathered}
\mathscr{L} V(t, e(t), p) \leq \sum_{i=1}^{N} e_{i}^{T}(t)\left\{\tilde{A}+\tilde{\Omega}+\left(\frac{1}{2} \Theta(r(t))+\tilde{O}-\Xi(r(t))\right) \otimes I_{n}\right\} e_{i}(t) \\
-\varphi(p)\left(\sum_{i=1}^{N} \frac{1}{\lambda_{i}(p)} \tilde{\alpha}_{i}^{T}(t) \tilde{\alpha}_{i}(t)\right)^{1+\gamma} \\
-k(p)\left(\sum_{i=1}^{N} e_{i}^{T}(t) e_{i}(t)\right)^{(1+\gamma) / 2} \\
-\xi\left(\sum_{i=1}^{N} \int_{t-\tau}^{t}\left(e_{i}^{T}(\theta) e_{i}(\theta)\right) d \theta\right)^{(1+\gamma) / 2} \\
+\sum_{q=1}^{m} \pi_{p q} \frac{1}{2} \sum_{i=1}^{N} e_{i}^{T}(t)(D(q)-Q(p)) e_{i}(t) \\
\leq \sum_{i=1}^{N} e_{i}^{T}(t)\left\{\widetilde{K}+\widetilde{A}+\tilde{\Omega}+\left(\frac{1}{2} \Theta(r(t))+\tilde{O}-\Xi(r(t))\right) \otimes I_{n}\right\} e_{i}(t) \\
-\psi\left(\sum_{i=1}^{N} e_{i}^{T}(t) e_{i}(t)+\sum_{i=1}^{N} \frac{1}{\lambda_{i}(p)} \tilde{\alpha}_{i}^{T}(t) \tilde{\alpha}_{i}(t)+2 \sum_{i=1}^{N} \int_{t-\tau}^{t} e_{i}^{T}(\theta) e_{i}(\theta) d \theta\right)
\end{gathered}
$$

According to conditions (17), the above inequality (30) 
can be simplified to

$$
\mathscr{L} V(t, e(t), p) \leq-\psi 2^{\varepsilon} V^{\varepsilon}(t, e(t), p),
$$

where $\varepsilon=(1+\gamma) / 2$.

From Lemma 4 and inequality (18), if $t_{0}=0$, finite time $t_{1}$ can be estimated as

$$
t_{1} \leq \frac{V(0, r(0))^{1-\varepsilon}}{\psi 2^{\varepsilon}(1-\varepsilon)} .
$$

Remark 10. When networks (7) and (11) achieve antisynchronization in a finite time $t_{1}$, the unknown parameter vector $\widehat{\alpha}_{i}(t)$ of the system will be identified as true value.

Remark 11. In the finite-time antisynchronization process between two nonlinear coupled double-weight Markovian switching complex networks (7) and (11), inequality (17) is only a sufficient condition, not a necessary condition.

Remark 12. The speed of antisynchronization and unknown parameters identification depends on the selection of control parameters $\eta_{i}(r), \lambda_{i}(r), k(r) \varphi(r)$ in controller (16).

Corollary 13. When the nonlinear coupling function $h(x)=x$ in complex networks, the systems (7) and (11) are linear coupled complex networks. Moreover, under the condition of Theorem 9, antisynchronization between two linear coupling multiple-weight Markovian switching complex networks can also achieved in a finite time $t_{1}$.

Under the condition of $h(x)=x$, the synchronization error system is as follows

$$
\begin{aligned}
\dot{e}_{i}(t)= & \dot{x}_{i}(t)+\dot{y}_{i}(t)=f_{i 1}\left(x_{i}(t)\right)+f_{i 2}\left(x_{i}(t)\right) \alpha_{i}(t)+f_{i 1}\left(y_{i}(t)\right) \\
& +f_{i 2}\left(y_{i}(t)\right) \widehat{\alpha}_{i}(t)+\sigma_{i}\left(t, e_{i}(t), r(t)\right) \dot{\omega}(t) \\
& +\sum_{j=1}^{N} c_{1} a_{i j}^{1}(r(t)) \Gamma_{1} x_{j}(t-\tau) \\
& +\sum_{j=1}^{N} c_{2} a_{i j}^{2}(r(t)) \Gamma_{2} x_{j}(t) \\
& +\sum_{j=1}^{N} c_{1} b_{i j}^{1}(r(t)) \Gamma_{1} y_{j}(t-\tau) \\
& +\sum_{j=1}^{N} c_{2} b_{i j}^{2}(r(t)) \Gamma_{2} y_{j}(t)+u_{i}(t)
\end{aligned}
$$

Theorem 14. Let $h(x)=x$ and Assumptions 1-3 hold. Then, networks (7) and (11) achieve antisynchronization under the set of controller (16) in finite time $t_{1}$, if the following conditions are satisfied:

(i) The following condition holds:

$$
\begin{gathered}
\sum_{q=1}^{m} \pi_{p q}(D(q)-Q(p)) \leq 0, \\
\widetilde{K}+\widetilde{A}+\tilde{\Omega}+\left(\frac{1}{2} \Theta(r(t))-\Xi(r(t))\right) \otimes I_{n} \leq 0,
\end{gathered}
$$

where $D(q)$ is a positive definite matrix of appropriate dimension, $Q(q)$ is an arbitrary symmetric matrix. $\Theta(r(t))=$ diag $\left\{\rho_{1}(r(t)), \rho_{2}(r(t)), \cdots, \rho_{N}(r(t))\right\} ; \quad \operatorname{sign}\left(e_{i}(t)\right)=\operatorname{diag}\{\operatorname{sign}($ $\left.e_{i 1}(t)\right)$, sign $\left.\left(e_{i 2}(t)\right), \cdots, \operatorname{sign}\left(e_{i n}(t)\right)\right\}$;

$$
\tilde{A}=(1 / 2)\left(A^{2 s}+\left(A^{2 s}\right)^{T}\right) ; \omega_{p}=\sum_{q=1}^{m}(D(q)-Q(p)) / 2 ; \widetilde{K}=
$$

$I_{N} \otimes \omega_{p} ; \quad \tilde{\Omega}=v I_{N} \otimes P, \quad \Xi(r(t))=\operatorname{diag}\left\{\eta_{1}(r(t)), \eta_{2}(r(t)), \cdots\right.$, $\left.\eta_{N}(r(t))\right\}$.

(ii) When the initial time $t_{0}=0, t_{1}$ is estimated as

$$
t_{1} \leq \frac{V(0, r(0))^{1-\varepsilon}}{\theta 2^{\varepsilon}(1-\varepsilon)},
$$

where $V\left(t_{0}, r\left(t_{0}\right)\right)=\left((1 / 2) \sum_{i=1}^{N} e_{i}^{T}\left(t_{0}\right) e_{i}\left(t_{0}\right)\right)+\left((1 / 2) \sum_{i=1}^{N}(1 /\right.$ $\left.\left.\lambda_{i}\left(r\left(t_{0}\right)\right)\right) \tilde{a}_{i}^{T}\left(t_{0}\right) \tilde{a}_{i}\left(t_{0}\right)\right), \theta=\min \{\min (k(r)), \min (\varphi(r))\}$.

Proof. Constructed Lyapunov function is the same as equation (19).

Therefore, under the condition of $h(x)=x$, inequality (23) is as follows

$$
\begin{aligned}
& \sum_{i=1}^{N} e_{i}^{T}(t) \sum_{j=1}^{N} c_{2} a_{i j}^{2}(p) \Gamma_{2} H\left(e_{j}(t)\right) \\
& \quad=\sum_{i=1}^{N} e_{i}^{T}(t) \sum_{j=1}^{N} c_{2} a_{i j}^{2}(p) \Gamma_{2} e_{j}(t) \\
& \quad=e^{T}(t)\left(A^{2} \otimes \Gamma_{2}\right) e(t) \leq \lambda_{\max }\left(\frac{A^{2 s}+\left(A^{2 s}\right)^{T}}{2}\right) e^{T}(t) e(t),
\end{aligned}
$$

where $A^{2 s}=\left(A^{2} \otimes \Gamma_{2}\right)$.

The rest of the proof is similar to that of Theorem 9 .

Remark 15. In the finite-time antisynchronization and parameters identification process between two linear coupled double-weight Markovian switching complex networks, inequality (34) is only a sufficient condition, not a necessary condition.

Corollary 16. When the unknown parameter vector $\alpha(t)$ of the network is known, under the condition of Theorem 9, complex networks (7) and (11) can achieve antisynchronization, where the adaptive part of the unknown parameter of the 
controller is cancelled in Theorem 9.

$$
\begin{aligned}
u_{i}(t)= & -k(r(t)) \operatorname{sign}\left(e_{i}(t)\right)\left|e_{i}(t)\right|^{\gamma}-\eta_{i}(r(t)) e_{i}(t) \\
& -\sum_{j=1}^{N} c_{1} a_{i j}^{1} r(t) \Gamma_{1} x_{j}(t-\tau)-\sum_{j=1}^{N} c_{1} b_{i j}^{1} r(t) \Gamma_{1} y_{j}(t-\tau) \\
& +\sum_{j=1}^{N} c_{2}\left(a_{i j}^{2} r(t)-b_{i j}^{2} r(t)\right) \Gamma_{2} h\left(y_{j}(t)\right) .
\end{aligned}
$$

Proof. The Lyapunov function is shown below

$$
V(t, e(t), p)=\frac{1}{2} \sum_{i=1}^{N} e_{i}^{T}(t) e_{i}(t)+\sum_{i=1}^{N} \int_{t-\tau}^{t} e_{i}^{T}(\theta) e_{i}(\theta) d \theta
$$

The proof process is basically similar to Theorem 9 .

Remark 17. Based on the synchronization control rules in this paper, a class of nonlinear coupled double-weight Markovian complex networks without unknown parameters can achieve finite-time antisynchronization.

Corollary 18. Under the conditions of Corollary 13, Corollary 16 , and $\Gamma_{1}=\Gamma_{2}$, complex networks (7) and (11) become singleweight Markovian complex networks. According to Theorem 9 and Theorem 14, finite-time antisynchronization between two linear coupled single-weight Markovian complex networks can be achieved. In addition, the proof process is basically similar to Theorem 9.

\section{Simulink Results}

In this section, we will give some typical numerical examples to illustrate the effectiveness of the above model analysis.

4.1. Example 1. Firstly, a three-dimensional network state equation is given, and the dynamics equations of each nodes can be described by a chaotic system.

$$
\left\{\begin{array}{l}
\dot{x}_{i 1}(t)=\alpha_{i 1}\left(x_{i 2}(t)-x_{i 1}(t)\right), \\
\dot{x}_{i 2}(t)=-x_{i 1}(t) x_{i 3}(t)+\alpha_{i 3} x_{i 2}(t), \\
\dot{x}_{i 3}(t)=x_{i 1}(t) x_{i 2}(t)-\alpha_{i 2} x_{i 3}(t) .
\end{array}\right.
$$

Both of the driving system and the response system can be expressed by the Chen system above, and the system

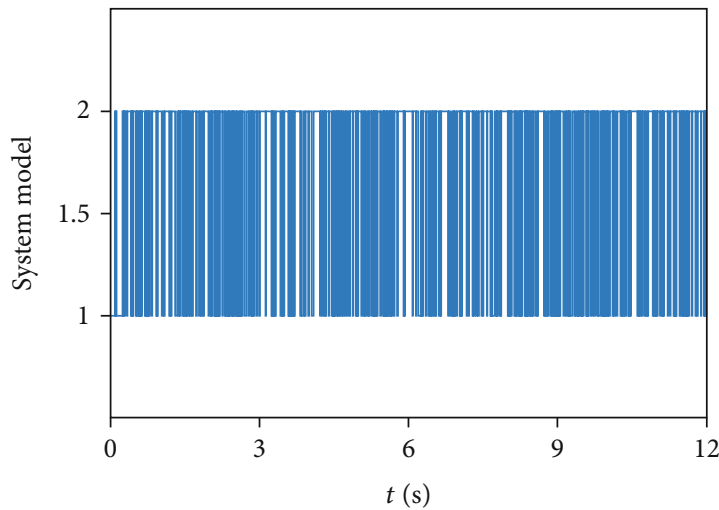

FIGURE 2: Switching of system mode.

(39) can be expressed as:

$$
\begin{aligned}
\left(\begin{array}{c}
\dot{x}_{i 1}(t) \\
\dot{x}_{i 2}(t) \\
\dot{x}_{i 3}(t)
\end{array}\right)= & \left(\begin{array}{c}
0 \\
-x_{i 1}(t) x_{i 3}(t) \\
x_{i 1}(t) x_{i 2}(t)
\end{array}\right) \\
& +\left(\begin{array}{ccc}
x_{i 2}(t)-x_{i 1}(t) & 0 & 0 \\
0 & 0 & x_{i 2}(t) \\
0 & -x_{i 3}(t) & 0
\end{array}\right) \times\left(\begin{array}{c}
\alpha_{i 1} \\
\alpha_{i 2} \\
\alpha_{i 3}
\end{array}\right),
\end{aligned}
$$

where $\alpha_{i}$ is an unknow parameter vector, and the identification value of the unknow parameter is $\alpha_{i}=(36,3,20)^{T}$. Moreover, the number of system nodes is 30 in numerical simulation, the coupling strengths of the networks are taken as $c_{1}=c_{2}=$ 10 , and the other relevant parameters are as follows.

Where $\quad h(x)=\sin (x), \quad \Pi=\left[\begin{array}{cc}2 & -2 \\ -1 & 1\end{array}\right], \quad \Gamma_{1}=$

$$
\begin{gathered}
{\left[\begin{array}{ccc}
0.5 & 0 & 0 \\
0 & 0.5 & 0 \\
0 & 0 & 0.5
\end{array}\right], \text { and } \Gamma_{2}=\left[\begin{array}{ccc}
1 & 0 & 0 \\
0 & 1 & 0 \\
0 & 0 & 1
\end{array}\right],} \\
\sigma_{i}\left(t, e_{i}(t), 1\right)=\operatorname{diag}\left(\frac{\sqrt{2}}{2} e_{i 1}, \frac{\sqrt{2}}{2} e_{i 2}, \frac{\sqrt{2}}{2} e_{i 3}\right), \\
\sigma_{i}\left(t, e_{i}(t), 2\right)=\operatorname{diag}\left(\sqrt{2} e_{i 1}, \sqrt{2} e_{i 2}, \sqrt{2} e_{i 3}\right) .
\end{gathered}
$$

The switching of network mode is shown in Figure 2. In the following, according to the controller (16) and condition (17), some control parameters of controller (16) are selected as follows: $\eta(1)=\eta(2)=40 ; k(1)=23, k(2)=25 ; \lambda(1)=20$, $\lambda(2)=25 ; \varphi(1)=\varphi(2)=10 ; \gamma=0.6 ; M_{\alpha}=50$. In this simulation process, the start time of the simulation is selected at $t_{0}$ $=0$, and time delay $\tau=0.01$. In addition, through Lemma 4 and (32), we can obtain synchronization time $t_{1} \leq 10.9854$. 

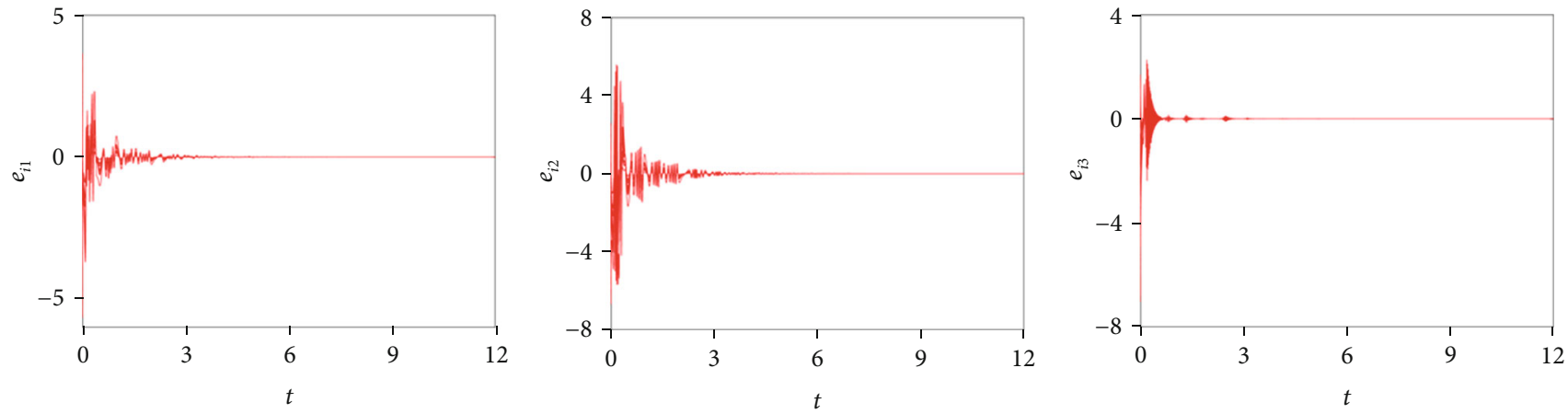

FIgURE 3: Synchronization errors $e_{i}(t)$ with system evolution.
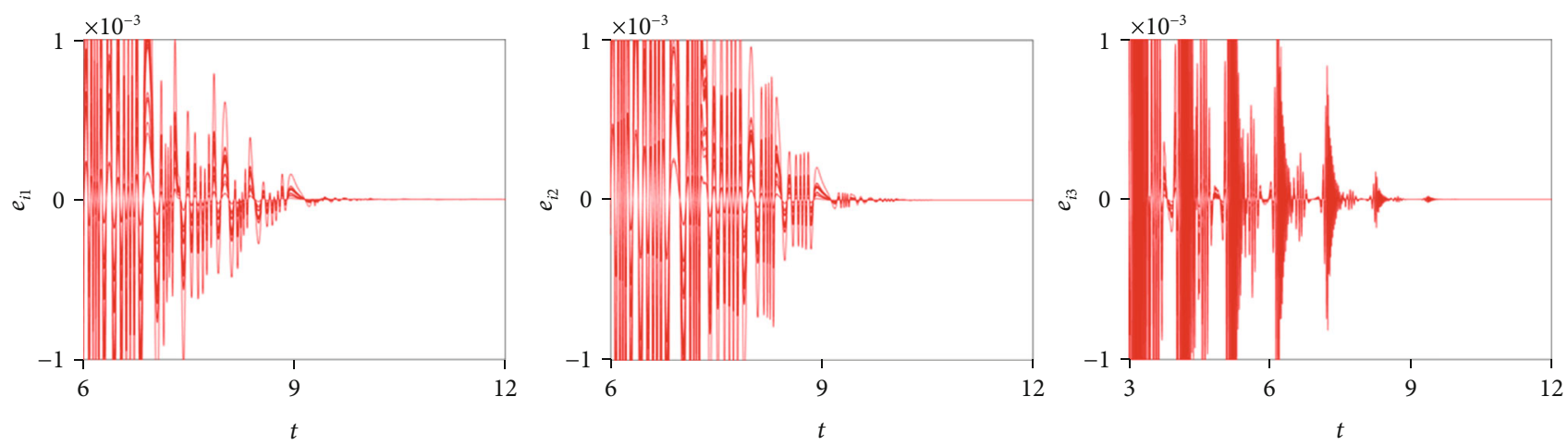

FIGURE 4: Partial amplification of synchronization errors $e_{i}(t)$.
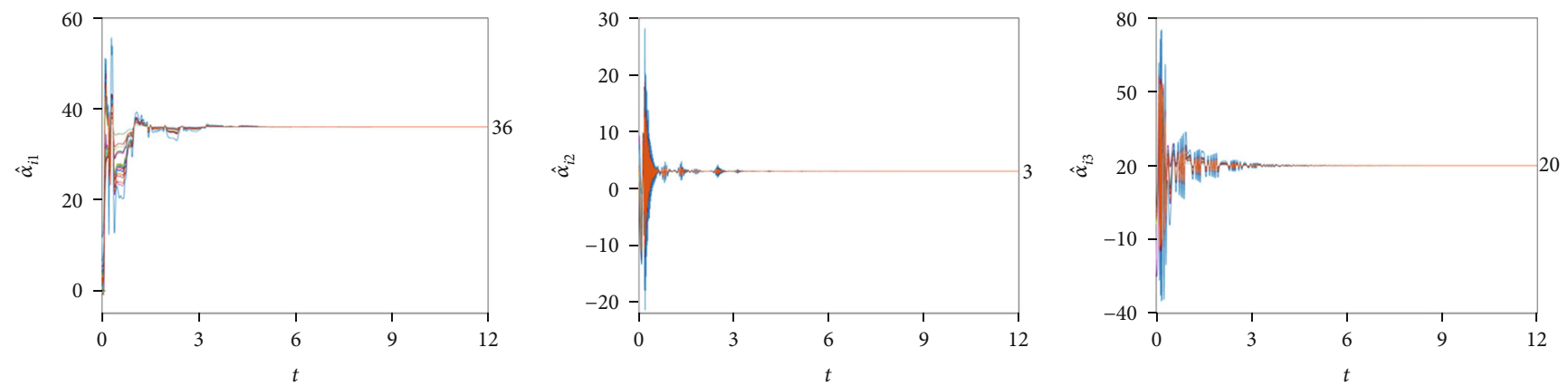

FIGURE 5: Identification of the uncertain parameters $\widehat{\alpha}_{i}$.
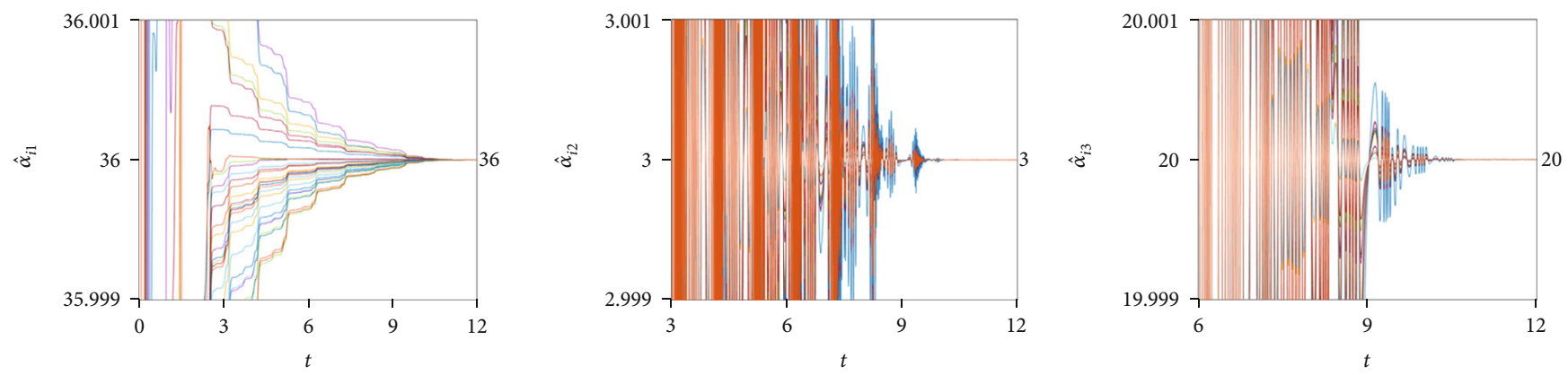

Figure 6: Partial amplification of the uncertain parameters $\widehat{\alpha}_{i}$. 
The time-varying curves of network synchronization errors $e_{i}(t)(i=1,2,3)$ are shown in Figure 3, and we can observe that synchronization error curves of networks gradually converge to zero. Furthermore, Figure 5 is the identification process of the unknown parameter vectors $\alpha_{i}(i=1,2,3)$, and the unknown parameter vector is finally identified as $\alpha_{i}=(36,3,20)^{T}$. From the enlarged pictures of Figures 4 and 6 , it can be clearly seen that the network synchronization and parameter identification are achieved at about $t=10$. By comparing calculated values with simulated values, the theoretical proof of the third part is also verified.

Secondly, in order to prove the superiority of this control method, the following comparison simulation is given, and the control method in [15] will be adopted. In addition, the network model, the topology switching mode, and all parameters are kept consistent in the two simulations. Figure 7 shows the system mode switching process.

In this simulation process, the control parameters of the network are basically the same as the above example. In addition, the start time of the simulation is selected at $t_{0}=0$, and time delay $\tau=0.01$. In addition, through Lemma 4 and (32), we can obtain synchronization time $t_{1} \leq 18.4358$.

The time-varying curves of networks synchronization errors $e_{i}(t)(i=1,2,3)$ are shown in Figure 8, and we can observe that synchronization error curves of networks gradually converge to zero. In addition, Figure 10 is the identification process of the unknown parameter vectors $\alpha_{i}(i=1,2,3)$, and the unknown parameter vector is finally identified as $\alpha_{i}$ $=(36,3,20)^{T}$. From the enlarged pictures of Figures 9 and 11 , it can be clearly seen that the networks synchronization and parameter identification are achieved at about $t=17$. By comparing calculated values with simulated values, the theoretical proof of the third part is also verified.

By comparing the results of Example 1, the following conclusions can be drawn. On the one hand, the antisynchronization and identification of the networks can be realized in a finite time. One the other hand, compared with the control strategy in [15], the synchronization and identification performance of the system can be realized faster under the control strategy proposed in this paper.

4.2. Example 2. In this subsection, in order to prove the wide applicability of this control method, a four-dimensional network state equation is given, and the dynamic equations of each node can be described by a Hyperchaotic system.

$$
\left\{\begin{array}{l}
\dot{x}_{i 1}(t)=\alpha_{i 1}\left(x_{i 2}(t)-x_{i 1}(t)\right), \\
\dot{x}_{i 2}(t)=-x_{i 1}(t) x_{i 3}(t)+\alpha_{i 3} x_{i 2}(t), \\
\dot{x}_{i 3}(t)=x_{i 1}(t) x_{i 2}(t)-\alpha_{i 2} x_{i 3}(t), \\
\dot{x}_{i 4}(t)=x_{i 1}(t) x_{i 3}(t)+\alpha_{i 4} x_{i 4}(t) .
\end{array}\right.
$$

Both of the driving system and the response system can be expressed by the Hyperchaotic system above, and the sys-

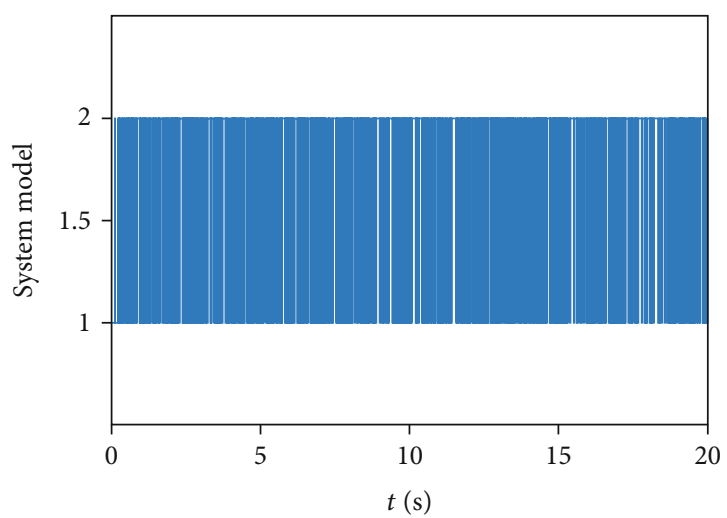

Figure 7: Switching of system mode.

tem (42) can be expressed as

$$
\begin{aligned}
\left(\begin{array}{c}
\dot{x}_{i 1} \\
\dot{x}_{i 4} \\
\dot{x}_{i 4} \\
\dot{x}_{i 4}
\end{array}\right) & \left(\begin{array}{c}
0 \\
-x_{i 1}(t) x_{i 3}(t) \\
x_{i 1}(t) x_{i 2}(t) \\
x_{i 1}(t) x_{i 3}(t)
\end{array}\right) \\
& +\left(\begin{array}{cccc}
x_{i 2}(t)-x_{i 1}(t) & 0 & 0 & x_{i 4}(t) \\
0 & 0 & x_{i 2}(t) & 0 \\
0 & -x_{i 3}(t) & 0 & 0 \\
0 & 0 & 0 & x_{i 4}(t)
\end{array}\right) \times\left(\begin{array}{c}
\alpha_{i 1} \\
\alpha_{i 2} \\
\alpha_{i 3} \\
\alpha_{i 4}
\end{array}\right),
\end{aligned}
$$

where $\alpha_{i}$ is an unknow parameter vector, and the identification value of the unknow parameter is $\alpha_{i}=(36,3,20,1)^{T}$. Moreover, the multiweighted Markovian switching network is composed of 100 nodes, the coupling strengths of the networks are taken as $c_{2}=c_{2}=10$, and the other relevant parameters are as follows.

$$
\text { Where } \quad h(x)=\cos (x), \quad \Pi=\left[\begin{array}{cc}
2 & -2 \\
-1 & 1
\end{array}\right], \quad \Gamma_{1}=
$$

$$
\begin{gathered}
{\left[\begin{array}{cccc}
1 & 0 & 0 & 0 \\
0 & 1 & 0 & 0 \\
0 & 0 & 1 & 0 \\
0 & 0 & 0 & 1
\end{array}\right], \text { and } \Gamma_{2}=\left[\begin{array}{cccc}
2 & 0 & 0 & 0 \\
0 & 2 & 0 & 0 \\
0 & 0 & 2 & 0 \\
0 & 0 & 0 & 2
\end{array}\right],} \\
\sigma_{i}\left(t, e_{i}(t), 1\right)=\operatorname{diag}\left(\frac{\sqrt{2}}{2} e_{i 1}, \frac{\sqrt{2}}{2} e_{i 2}, \frac{\sqrt{2}}{2} e_{i 3}\right), \\
\sigma_{i}\left(t, e_{i}(t), 2\right)=\operatorname{diag}\left(\sqrt{2} e_{i 1}, \sqrt{2} e_{i 2}, \sqrt{2} e_{i 3}\right) .
\end{gathered}
$$

The switching of network mode is shown in Figure 7. In the following, some control parameters of controller (16) 

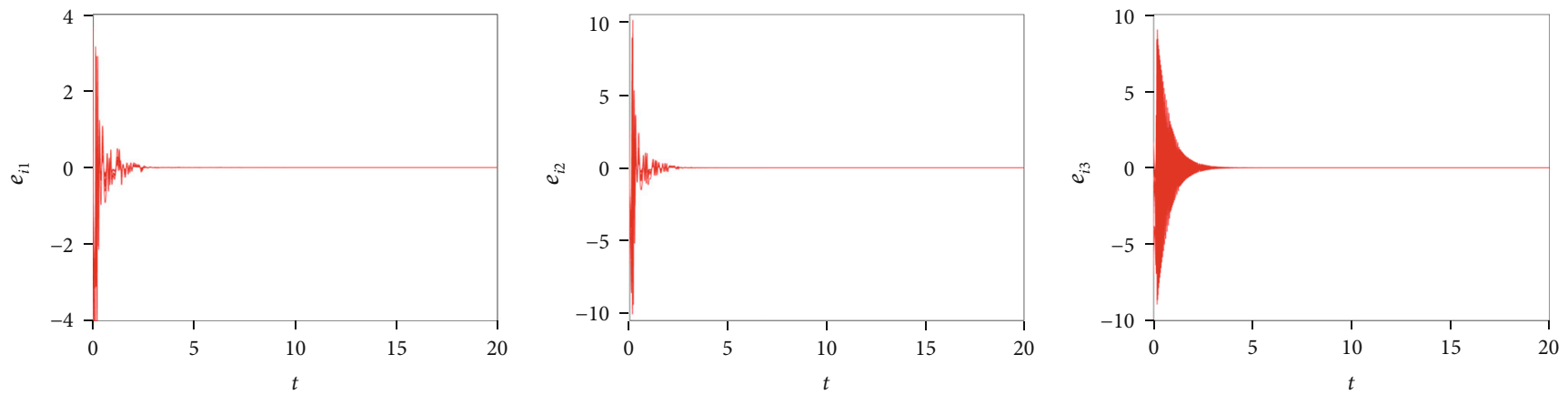

FIgURE 8: Synchronization errors $e_{i}(t)$ with system evolution.
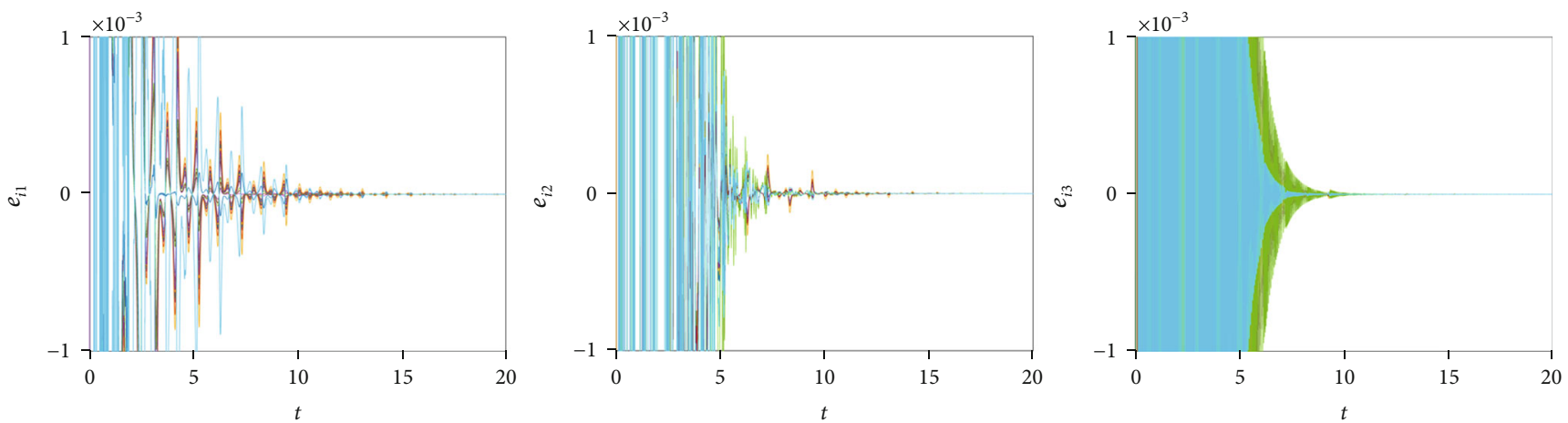

Figure 9: Partial amplification of synchronization errors $e_{i}(t)$.
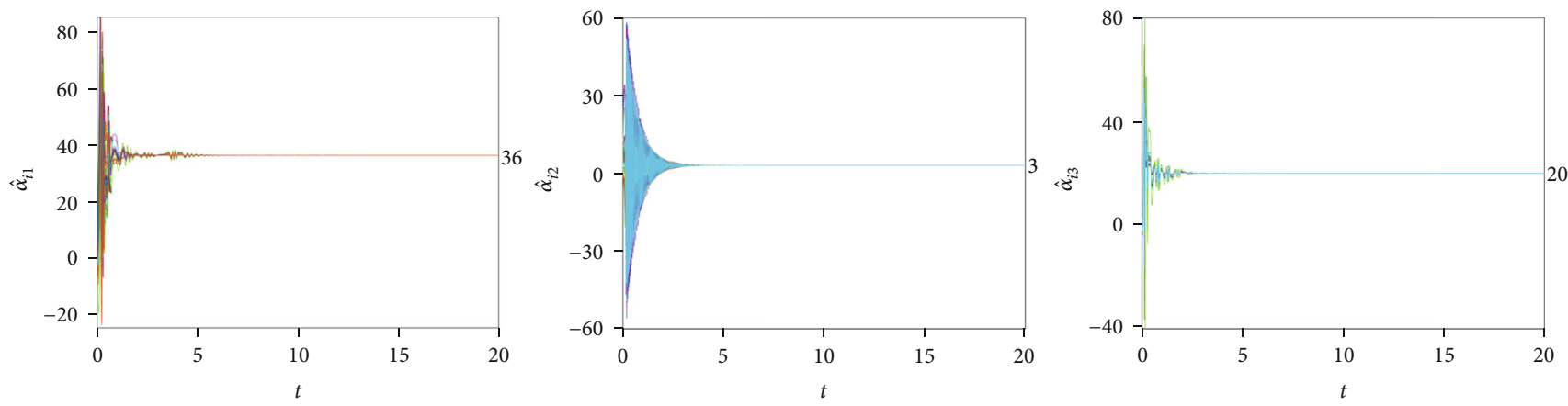

Figure 10: Identification of the uncertain parameters $\widehat{\alpha}_{i}$.
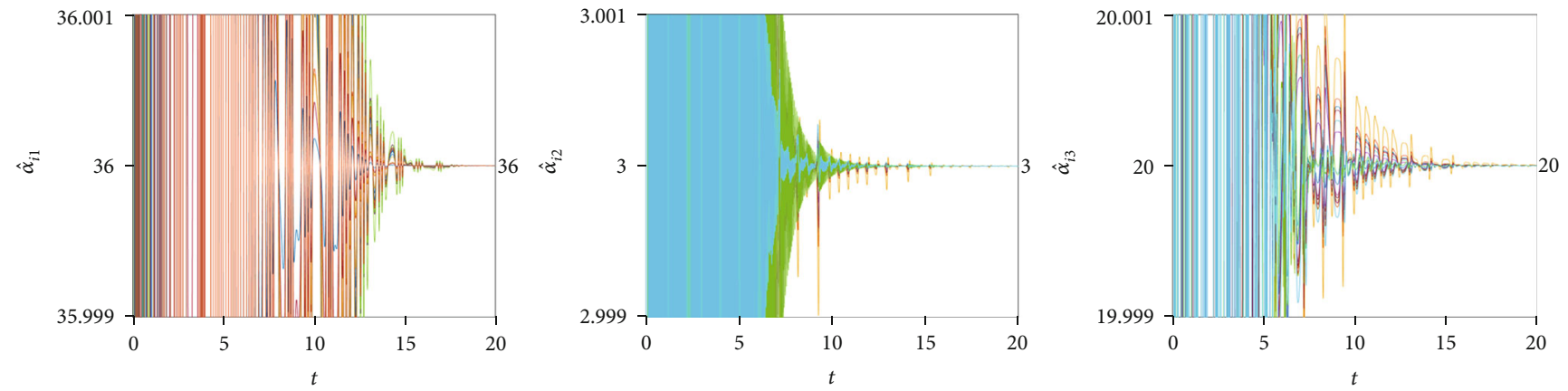

FIGURE 11: Partial amplification of the uncertain parameters $\widehat{\alpha}_{i}$. 

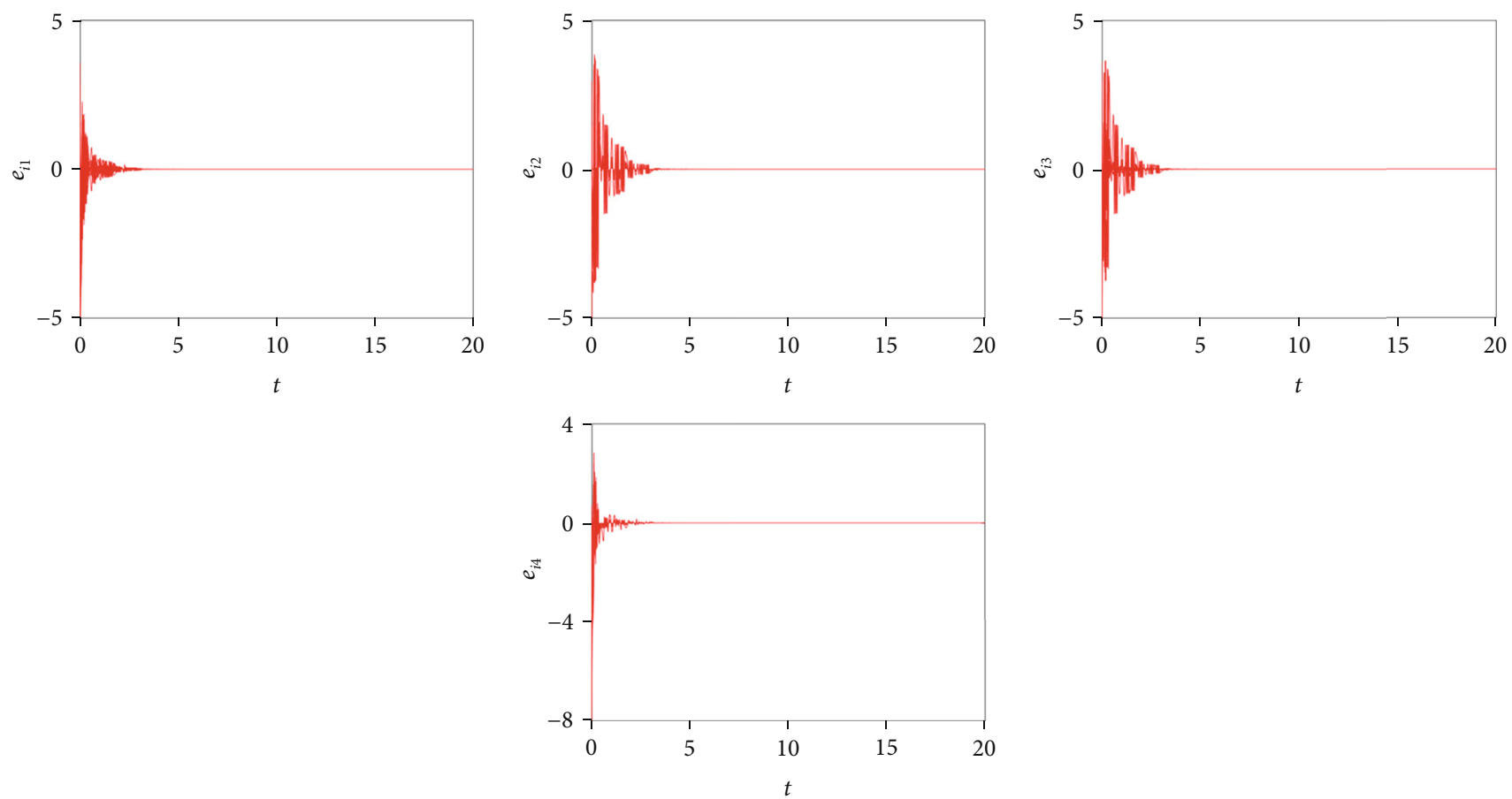

FIGURE 12: Synchronization errors $e_{i}(t)$ with system evolution.
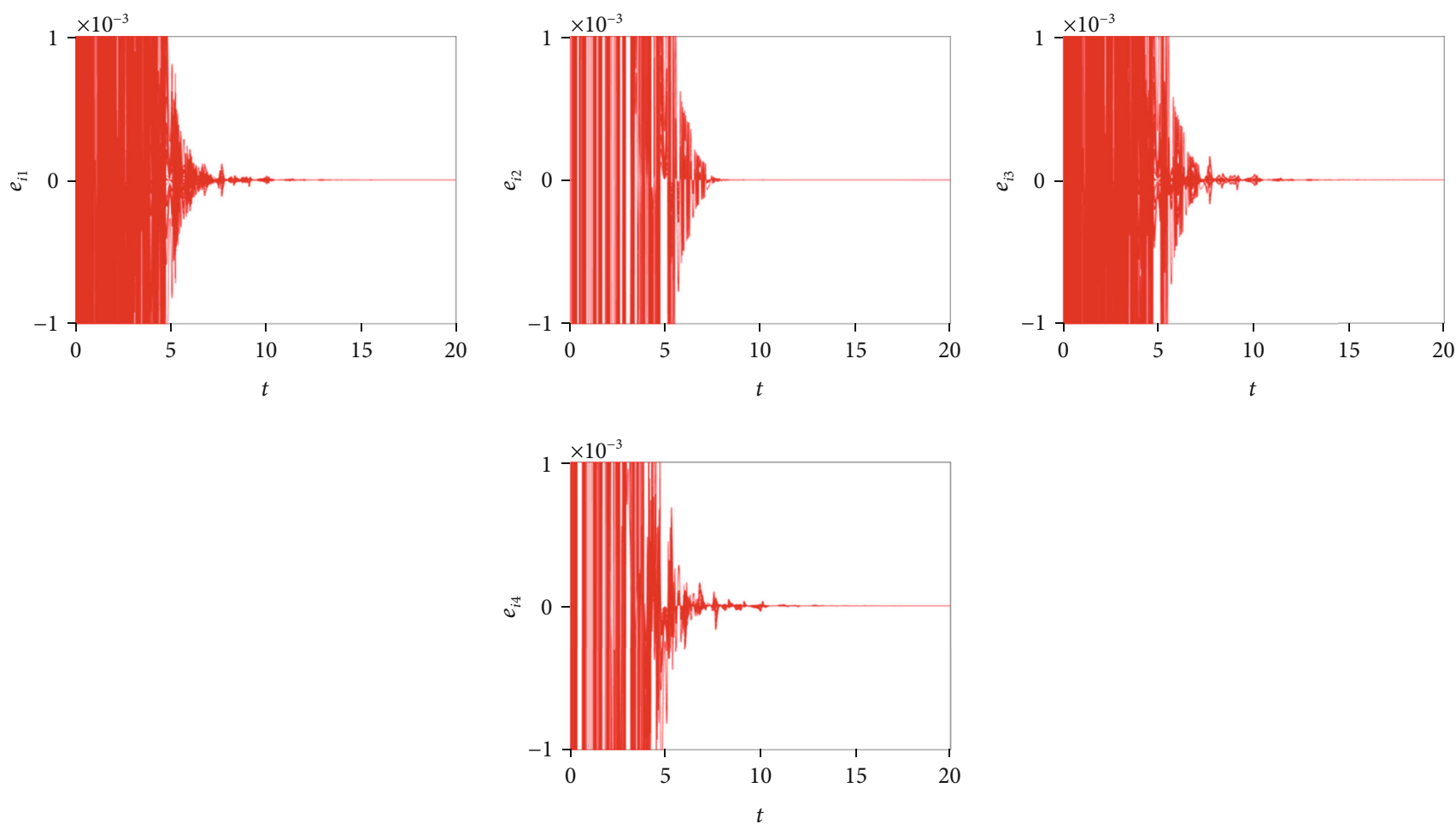

FIgURE 13: Partial amplification of synchronization errors $e_{i}(t)$.

are selected as follows: $\eta(1)=\eta(2)=32 ; k(1)=20, k(2)=18$; $\lambda(1)=18, \lambda(2)=25 ; \varphi(1)=\varphi(2)=9 ; \gamma=0.6 ; M_{\alpha}=50$. In this simulation process, the start time of the simulation is selected at $t_{0}=0$, and time delay $\tau=0.01$. In addition, through Lemma 4 and (32), we can obtain synchronization time $t_{1} \leq 18.4391$.
The time-varying curves of networks synchronization errors $e_{i}(t)(i=1,2,3,4)$ are shown in Figure 12, and we can observe that synchronization error curves of networks gradually converge to zero. Furthermore, Figure 14 is the identification process of the unknown parameter vectors $\alpha_{i}(i=1,2,3,4)$, and the unknown parameter vector is 

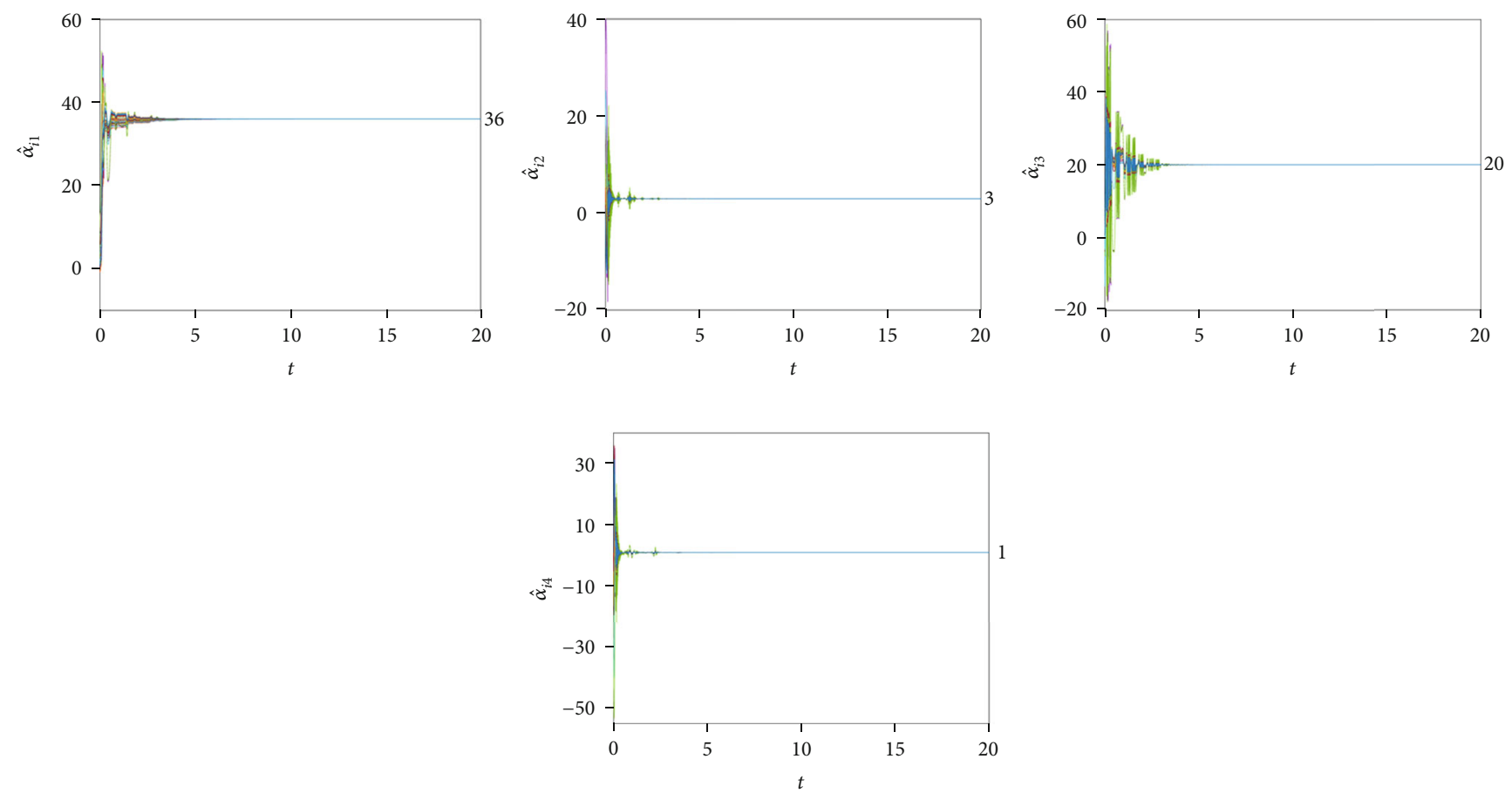

FIgURE 14: Identification of the uncertain parameters $\widehat{\alpha}_{i}$.
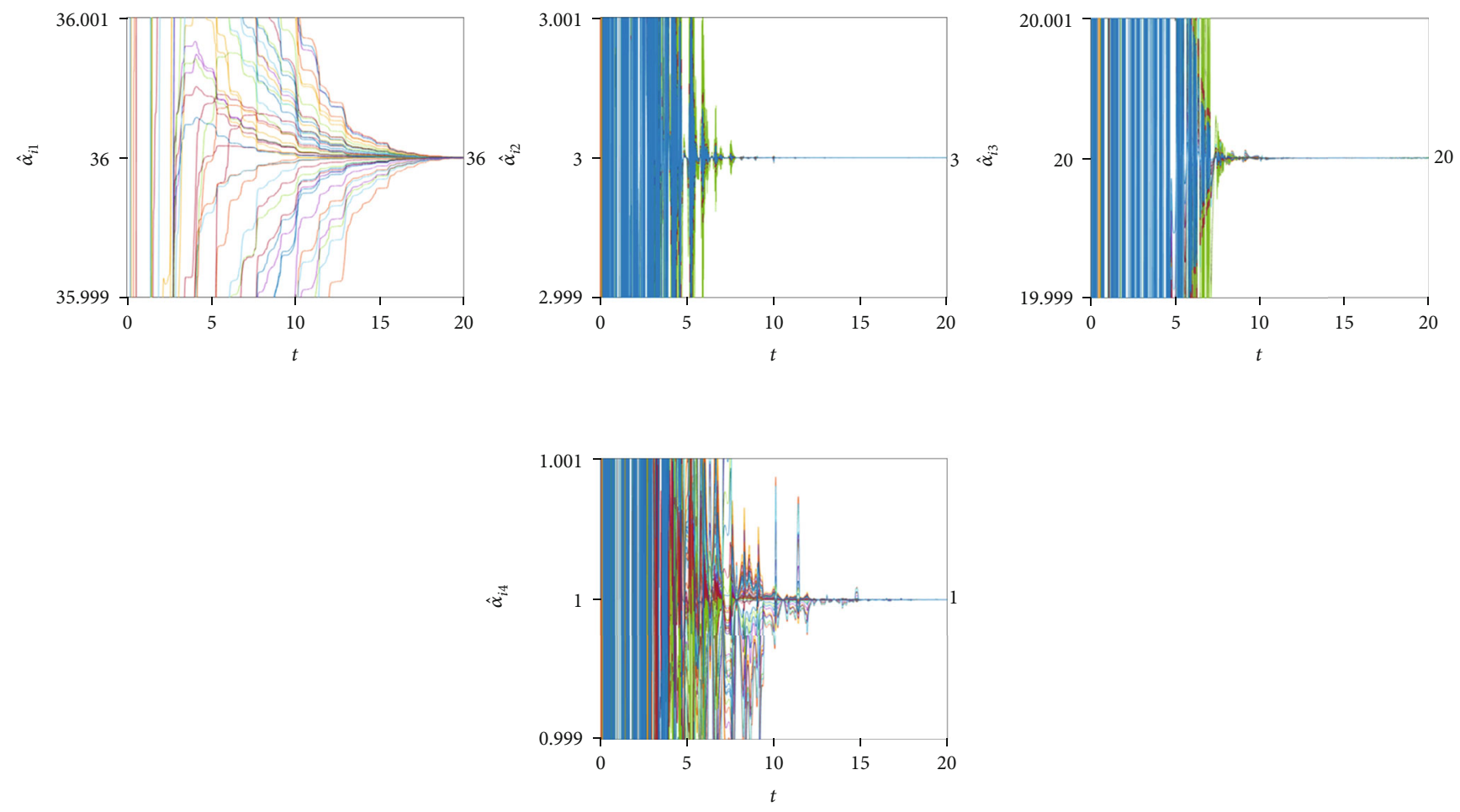

FIgURE 15: Partial amplification of the uncertain parameters $\widehat{\alpha}_{i}$.

finally identified as $\alpha_{i}=(36,3,20,1)^{T}$. From the enlarged pictures of Figures 13 and 15, it can be clearly seen that the networks synchronization and parameter identification are achieved at about $t=16$. By comparing calculated values with simulated values, the theoretical proof of the third part is also verified.

\section{Conclusion}

In this paper, the finite-time antisynchronization and parameter identification of a class of nonlinear coupled multiple-weight Markovian switching complex networks with stochastic disturbances is solved. Firstly, by a controller 
with an adaptive law, sufficient and unnecessary conditions for synchronization of nonlinear coupling networks are obtained, and at the same time, unknown parameters of networks are identified. Secondly, several corollaries show that the synchronization control method in this paper can be applied to more general complex networks. Finally, simulation results prove that network models can achieve antisynchronization in a finite time, and the unknown parameter vector of networks is also identified as true values.

In the future, we will continue to study the following aspects. On the one hand, it is very challenging to realize the finite-time antisynchronization and parameters identification of the proposed models by controlling a small number of important nodes. On the other hand, based on the model proposed in this paper, it is also very interesting to study the nonlinear coupled multiple-weight Markovian switching complex network models with parameter and model uncertainties and time-varying delays.

\section{Data Availability}

The data used to support the findings of this study are included within the article.

\section{Conflicts of Interest}

The authors declare that they have no competing interests.

\section{Authors' Contributions}

Each author has made equal contributions to the research and writing of this article. The author read and approved the final manuscript.

\section{References}

[1] Y. Wu, Y. Li, and W. Li, "Synchronization of random coupling delayed complex networks with random and adaptive coupling strength," Nonlinear Dynamics, vol. 96, no. 4, pp. 2393-2412, 2019.

[2] K. Wu, B. Li, Y. Du, and S. Du, "Synchronization for impulsive hybrid-coupled reaction-diffusion neural networks with timevarying delays," Communications in Nonlinear Science and Numerical Simulation, vol. 82, article 105031, 2020.

[3] Z. Hao, W. Xing-yuan, Y. Peng-fei, and S. Yu-jie, "Combination synchronization and stability analysis of time-varying complex-valued neural networks," Chaos, Solitons \& Fractals, vol. 131, article 109485, 2020.

[4] H. Zhang and X.-y. Wang, "Complex projective synchronization of complex-valued neural network with structure identification," Journal of the Franklin Institute, vol. 354, no. 12, pp. 5011-5025, 2017.

[5] J.-L. Wang, P.-C. Wei, H.-N. Wu, T. Huang, and M. Xu, "Pinning synchronization of complex dynamical networks with multiweights," IEEE Transactions on Systems, Man, and Cybernetics: Systems, vol. 49, no. 7, pp. 1357-1370, 2019.

[6] C. Zhang, X. Wang, C. Wang, and Z. Liu, "Synchronization in nonlinear complex networks with multiple time-varying delays via adaptive aperiodically intermittent control," Inter- national Journal of Adaptive Control and Signal Processing, vol. 33, no. 1, pp. 39-51, 2019.

[7] Y. Liang, X. Wang, and J. Eustace, “Adaptive synchronization in complex networks with non-delay and variable delay couplings via pinning control," Neurocomputing, vol. 123, pp. 292-298, 2014.

[8] F. Nian and X. Wang, "Optimal pinning synchronization on directed complex network," Chaos, vol. 21, no. 4, article 043131, 2011.

[9] X.-Y. Wang and X.-W. Liu, "A new pinning control scheme of complex networks based on data flow," Nonlinear Dynamics, vol. 92, no. 1, pp. 13-24, 2018.

[10] Z. Hao, W. Xing-yuan, and L. Xiao-hui, "Synchronization of complex-valued neural network with sliding mode control," Journal of the Franklin Institute, vol. 353, no. 2, pp. 345-358, 2016.

[11] Y. Liang and X. Wang, "Synchronization in complex networks with non-delay and delay couplings via intermittent control with two switched periods," Physica A: Statistical Mechanics and its Applications, vol. 395, pp. 434-444, 2014.

[12] Z. Wang and X. Liu, "Synchronization of interconnected discontinuous neural networks with nonlinear coupling functions," IEEE Access, vol. 7, pp. 25804-25814, 2019.

[13] C. Yi, J. Feng, J. Wang, C. Xu, Y. Zhao, and Y. Gu, "Pinning synchronization of nonlinear and delayed coupled neural networks with multi-weights via aperiodically intermittent control," Neural Processing Letters, vol. 49, no. 1, pp. 141-157, 2019.

[14] T. Chen, S. Peng, and Z. Zhang, "Finite-time synchronization of Markovian jumping complex networks with non-identical nodes and impulsive effects," Entropy, vol. 21, no. 8, p. 779, 2019.

[15] X. Wang, K. Gao, G. Chen, and Y. Xu, "Finite-time synchronization control relationship analysis for two classes of Markovian jump complex networks under feedback control," Advances in Difference Equations, vol. 2018, no. 1, Article ID $382,2018$.

[16] Q. Liu, B. Kan, Q. Wang, and Y. Fang, "Cluster synchronization of Markovian switching complex networks with hybrid couplings and stochastic perturbations," Physica A: Statistical Mechanics and Its Applications, vol. 526, article 120937, 2019.

[17] H.-L. Li, J. Cao, H. Jiang, and A. Alsaedi, "Finite-time synchronization and parameter identification of uncertain fractionalorder complex networks," Physica A: Statistical Mechanics and its Applications, vol. 533, article 122027, 2019.

[18] X.-1. An, L. Zhang, and J.-g. Zhang, "Research on urban public traffic network with multi-weights based on single bus transfer junction," Physica A: Statistical Mechanics and its Applications, vol. 436, pp. 748-755, 2015.

[19] X.-1. An, L. Zhang, Y.-z. Li, and J.-g. Zhang, "Synchronization analysis of complex networks with multi-weights and its application in public traffic network," Physica A: Statistical Mechanics and its Applications, vol. 412, pp. 149-156, 2014.

[20] J. Wang, T. Ru, J. Xia, Y. Wei, and Z. Wang, "Finite-time synchronization for complex dynamic networks with semiMarkov switching topologies: an $H^{\infty}$ event-triggered control scheme," Applied Mathematics and Computation, vol. 356, pp. 235-251, 2019.

[21] X. Wang, B. Yang, K. Gao, and J.-a. Fang, "Finite-time synchronization control relationship analysis of two classes of Markovian switched complex networks," International 
Journal of Control Automation and Systems, vol. 16, no. 6, pp. 2845-2858, 2018.

[22] K. S. Anand, G. A. Harish Babu, M. Syed Ali, and S. Padmanabhan, "Finite-time synchronization of Markovian jumping complex dynamical networks and hybrid couplings," Chinese Journal of Physics, vol. 62, pp. 304-312, 2019.

[23] Y. Wang, H. Zhang, X. Wang, and D. Yang, "Networked synchronization control of coupled dynamic networks with timevarying delay," IEEE Transactions on Systems, Man, and Cybernetics, Part B (Cybernetics), vol. 40, no. 6, pp. 14681479, 2010.

[24] C. Zhang, X. Wang, S. Unar, and Y. Wang, "Finite-time synchronization of a class of nonlinear complex-valued networks with time-varying delays," Physica A: Statistical Mechanics and Its Applications, vol. 528, article 120985, 2019.

[25] Y. Sun, W. Li, and D. Zhao, "Finite-time stochastic outer synchronization between two complex dynamical networks with different topologies," Chaos, vol. 22, no. 2, article 023152, 2012.

[26] J. Yin, S. Khoo, Z. Man, and X. Yu, "Finite-time stability and instability of stochastic nonlinear systems," Automatica, vol. 47, no. 12, pp. 2671-2677, 2011.

[27] S. Boyd, L. el Ghaoui, E. Feron, and V. Balakrishnan, Linear Matrix Inequalities in System and Control Theory, SIAM, Philadelphia, 1994.

[28] J. Mei, M. Jiang, W. Xu, and B. Wang, "Finite-time synchronization control of complex dynamical networks with time delay," Communications in Nonlinear Science and Numerical Simulation, vol. 18, no. 9, pp. 2462-2478, 2013.

[29] C. Yuan and X. Mao, "Robust stability and controllability of stochastic differential delay equations with Markovian switching," Automatica, vol. 40, no. 3, pp. 343-354, 2004. 